EPJ Web of Conferences 102, 00002 (2015)

DOI: $10.1051 /$ epjconf/ 201510200002

(C) Owned by the authors, published by EDP Sciences, 2015

\title{
Disk formation and structure ${ }^{\star}$
}

\author{
Carsten Dominik \\ ${ }^{1}$ Anton Pannekoek Institute for Astronomy, University of Amsterdam, The Netherlands
}

\begin{abstract}
In this chapter, we cover the formation of protoplanetary disk as a natural byproduct of the gravitational collapse of a slowly rotating, hydrostatic cloud. We also summarise the structure of such a disk as it results from hydrostatic equilibrium and viscous accretion.
\end{abstract}

\section{Introduction}

In this chapter we look at the basic processes disk formation and of disk structure. Why do disks form in the first place? How will matter be distributed, radially and vertically? We will focus mainly on the structure of disks resulting from viscous evolution. Another important process affecting disk structure is external irradiation. As this is so strongly coupled with the spectral energy distribution we observe from protoplanetary disks, we will cover this part in the chapter on spectral energy distributions. The original idea of a protosolar nebula dates back to Kant \& Laplace in the 18th century who used the observed structure of the planetary system to propose the existence of a flattened structure around the Sun from which the planets formed, explaining both the flattened geometry and the aligned angular momenta of the Sun and the planetary orbits (nothing was known back then about planetary rotation).

The confirmation that such disks indeed exist and that they are a common by-product of star formation dates back to the late 1980's and early 1990's, when the first mm images (Beckwith et al. 1986, Sargent \& Beckwith 1987, Rodriguez et al. 1992) and spectroscopy (e.g. Koerner et al. 1993) of such disks were taken (Fig. 1). The mm dust emission revealed the extended structures around young stars and the double peaked line profiles were proof of the regular Keplerian rotation pattern. The launch of the Hubble Space Telescope in 1990 opened a new window for research on protoplanetary disks. The high spatial resolution from space enabled the detailed imaging of the Orion nebula, a star forming region at a distance of $450 \mathrm{pc}$ (O'Dell et al. 1992). These images were taken with the Wide Field Camera in several optical narrow band Filters such as $\mathrm{H} \alpha$, [O III], [O I] and [S II]. The data shows not only that such protoplanetary disks are ubiquitous around newly formed stars $(50 \%$ of stars show disk detection), but reveals also the impact of disk irradiation and erosion by nearby hot $\mathrm{O}$ and $\mathrm{B}$ stars.

\section{Disk formation as a result of the collapse of a rotating cloud}

Protoplanetary disks are the natural byproduct of the formation of a star from a rotating cloud of gas. For the purpose of this chapter we are not concerned how exactly the details of the collapse process

^First Lecture of the Summer School "Protoplanetary Disks: Theory and Modelling Meet Observations"

This is an Open Access article distributed under the terms of the Creative Commons Attribution License 4.0, which permits unrestricted use, distribution, and reproduction in any medium, provided the original work is properly cited. 

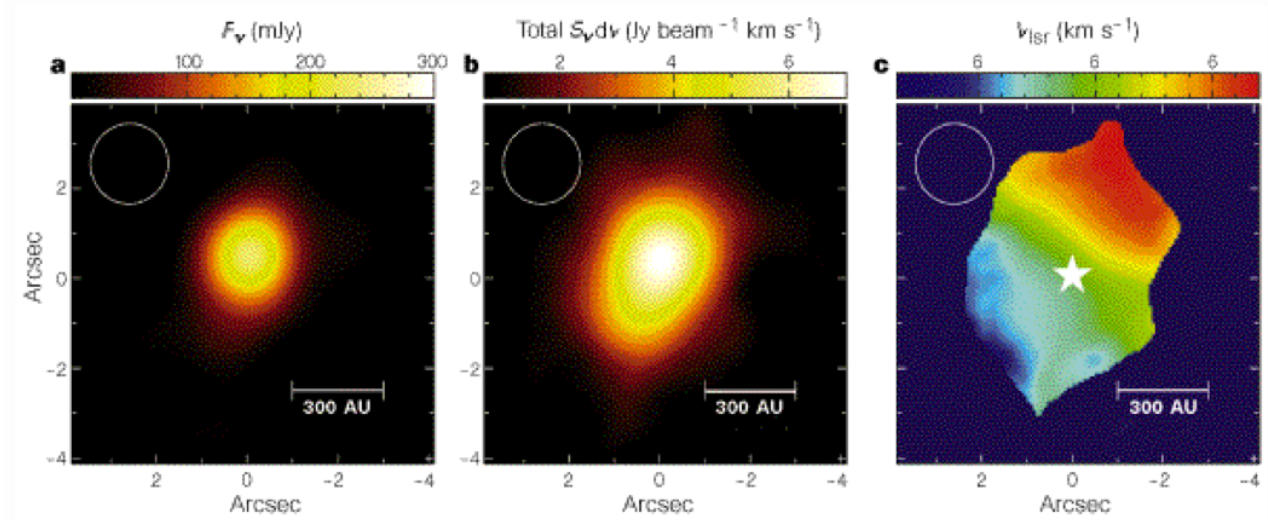

Figure 1. In each panel, the full-width at half-maximum of the synthesised beam is shown at top left, and offsets in arcseconds from the stellar position are indicated along the vertical and horizontal axes. (a) Thermal continuum from dust grains, at wavelength $\lambda=1.3 \mathrm{~mm}$. The flux density $(F)$ scale is shown at the top of the panel. (b) $\mathrm{CO}(2-$ 1) emission, integrated across the full velocity range $\left(+2.4\right.$ to $\left.+7.6 \mathrm{~km} \mathrm{~s}^{-1}\right)$ in which emission is measured above the $3 \sigma$ level. The scale at the top of the panel provides the integrated intensities $(S d v)$. (c) Intensity-weighted mean gas velocities at each spatial point of the structure shown in $b$. The stellar (systemic) velocity, $V_{\mathrm{lsr}} \approx$ $5.1 \mathrm{~km} \mathrm{~s}^{-1}$, is represented by green. Blue-shifted (approaching) and red-shifted (receding) velocity components are shown as blue and red, respectively. The star symbol indicates the position of MWC480. Reprinted by permission from Macmillan Publishers Ltd: Nature (Mannings et al. 1997), copyright (1997).

work - there are a number of competing theories that describe this phase. One of them is the inside-out collapse model (Shu 1977), but in other scenarios the entire cloud starts collapsing at the same time. What we will consider here is how the fact that a cloud is rotating ever so slowly before the collapse will lead to the formation of an accretion disk.

We assume that the cloud was in hydrostatic equilibrium for some time before collapse, and we will therefore assume that it rotates as a solid body. Also, we assume that the cloud rotates slowly, so that the centrifugal force is initially very small compared to the gravity and pressure forces at any point in the cloud. Then, the early stages of collapse are almost radial. In this limit, we can distinguish between an outer envelope which stays almost spherical and an inner region that gets distorted due to rotation and forms an equatorial accretion disk.

Depending on the specific angular momentum (that is the angular momentum per unit of mass) of a gas parcel, it will either be able to accrete onto the protostellar core (very low specific angular momentum) or accrete onto the equatorial disk at some radius corresponding to its angular momentum. A particle initially located on the rotation axis of the cloud has zero angular momentum and will simply fall directly to the center, i.e. the star. The maximum possible distance from the star at which such a gas parcel can end up is called the centrifugal radius $r_{c}$, and it is reached by particles that are initially located in the midplane of the system, i.e. the plane perpendicular to the rotation axis.

If we study the trajectory of a fluid element during the infall, we can assume that the protostar and its disk are a point mass, so no higher moments of the gravity field have to be considered. Also the mass in the outer cloud is centrally symmetric, and we ignore the mass in the transition region. Under these circumstances, the fluid element will have a bound elliptical orbit. However, since the initial energy is small compared to the energy it has when it reaches the equatorial plane, we can 

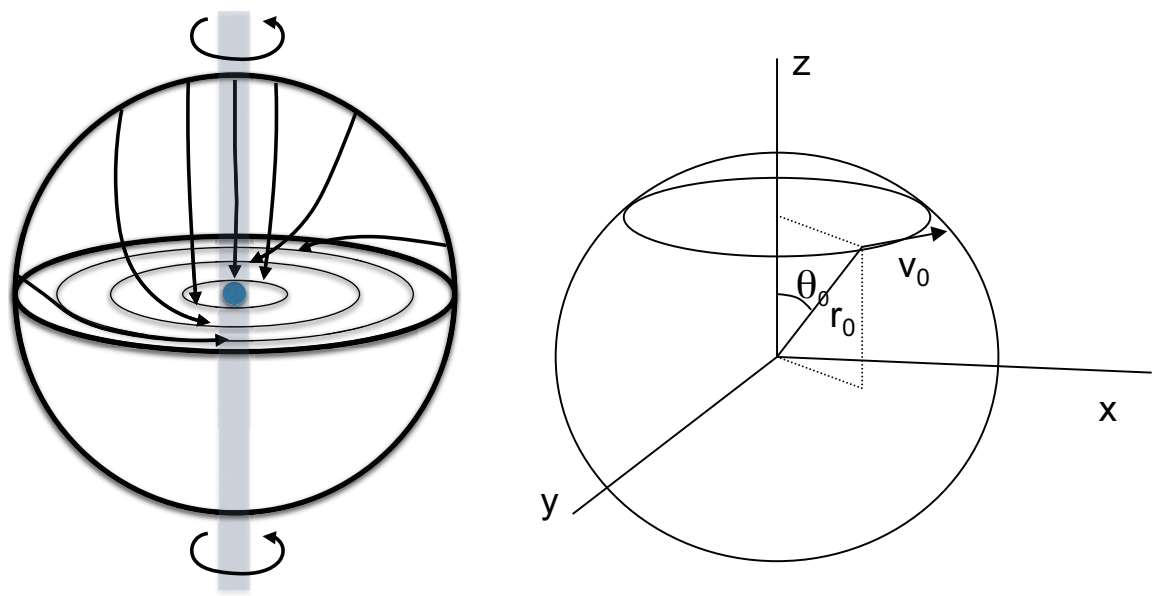

Figure 2. Sketch of a collapse including rotation. Left panel: A rotating cloud collapses, and different parcels fall according to their specific angular momentum along a path associated with their angular momentum. Right panel: Definition of the geometric quantities describing the initial position and velocity of the fluid element before falling.

approximate the orbit quite well by a parabola with focus at the center of the cloud. The distance from the star can then be written as a function of the true anomaly using Kepler's equation for the parabola

$$
r=\frac{r_{\mathrm{eq}}}{1+\cos \Psi},
$$

where the coordinates and quantities are introduced in Fig. 3. If the fluid element would reach the closest distance $r_{\min }$, the maximum orbital velocity would at that point be perpendicular to the direction to the star and have the value

$$
v_{\max }=\sqrt{\frac{2 G M_{*}}{r_{\min }}},
$$

where $M_{*}$ is the mass of the protostar + disk. Note that $M_{*}$ may be a function of time during star formation, so fluid elements that fall early on experience a different gravitational potential than fluid elements that fall late.

The fluid element will never reach the minimum stellar distance though. Instead, it collides with the forming disk and with matter coming in on a similar trajectory from the other side of the midplane at $\Psi=\pi / 2$ (see Fig. 4). The specific angular momentum $j$ is preserved along the entire curve and most easily computed at the theoretical minimum distance because radius vector and velocity are perpendicular. There we find

$$
j=r_{\min } v_{\max }=\sqrt{2 G M_{*} r_{\min }} .
$$

Since the fluid element reaches $r_{\min }$ at $\Psi=0$, Eq. (1) implies that $r_{\min }=r_{\text {eq }} / 2$. Therefore, we can write the radius at which the fluid element reaches the midplane as

$$
r_{\mathrm{eq}}=\frac{j^{2}}{G M_{*}} \text {. }
$$




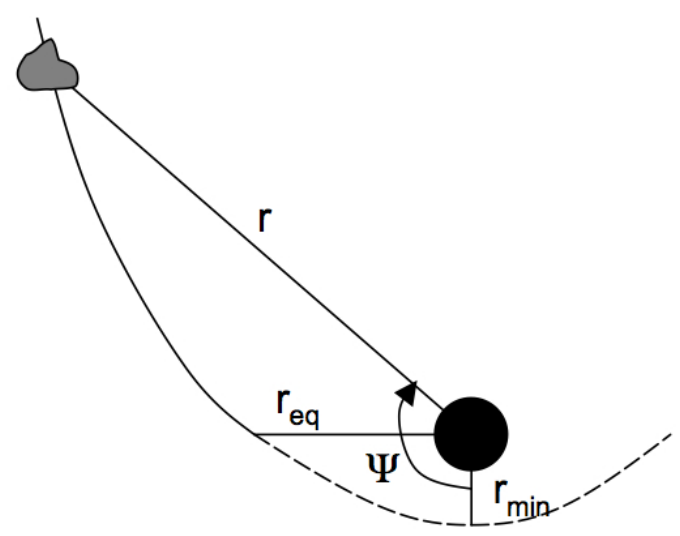

Figure 3. Sketch of a parabolic orbit in rotating infall. The sketch is in the orbital plane of the falling fluid element. A fluid element with polar coordinates $(r, \Psi)$ falls in and hits the equatorial disk at a radius $r_{\text {eq }}$. In the absence of the disk, the fluid element would have reached the smaller distance $r_{\min }$ before swinging back out again.

Now we can express the specific angular momentum in terms of the initial quantities $\Omega_{0}$ (angular velocity) and $\theta_{0}$ (inclination of orbital plane with respect to the rotation axis, $\theta_{0}=90^{\circ}$ is the midplane, see Fig. 2)

$$
j=R^{2} \Omega_{0} \sin \theta_{0},
$$

where $R$ is the distance from the center at which the fluid element starts its fall. We can see that fluid elements from larger distances will have larger specific angular momentum and will therefore become part of the disk at larger and larger distances from the star. In the Shu inside-out collapse (Shu 1977), that layer starts to fall when the rarefaction wave that starts in the center of the cloud at $t=0$, reaches $R$, i.e. when it has travelled with the isothermal sound speed $c_{s}$ to that location. Then, $R=c_{s} t$, and we can write

$$
j=R^{2} \Omega_{0} \sin \theta_{0}=c_{s}^{2} t^{2} \Omega_{0} \sin \theta_{0}
$$

The angular momentum is larger for higher values of $\theta_{0}$ and has a maximum at $\theta_{0}=90^{\circ}$. Using the expression for the core mass as a function of time $M_{r}(t) \simeq c_{s}^{3} t / G$ from Shu's model (Shu 1977), we can rewrite $r_{\mathrm{eq}}$ as

$$
r_{\mathrm{eq}}=c_{s} t^{3} \Omega_{0}^{2} \sin \theta_{0}^{2} \text {. }
$$

For $\theta_{0}=90^{\circ}, r_{\mathrm{eq}}$ is maximal, and we call this value the centrifugal radius of the disk

$$
r_{c}=c_{s} t^{3} \Omega_{0} \approx 7.33 \mathrm{AU}\left(\frac{c_{s}}{0.35 \mathrm{~km} / \mathrm{s}}\right)\left(\frac{\Omega}{10^{-14} \mathrm{~s}^{-1}}\right)^{2}\left(\frac{t}{10^{5} \mathrm{yr}}\right)^{3} .
$$

The value $\Omega=10^{-14} \mathrm{~s}^{-1}$ is close to typical observed values of a few times $10^{-14}-10^{-13} \mathrm{~s}^{-1}$. This gives us also an estimate of the initial size of the accretion disk. We will later see that the size of this initial disk grows in the later stages of star formation due to e.g. angular momentum transport (viscous spreading of the disk). 


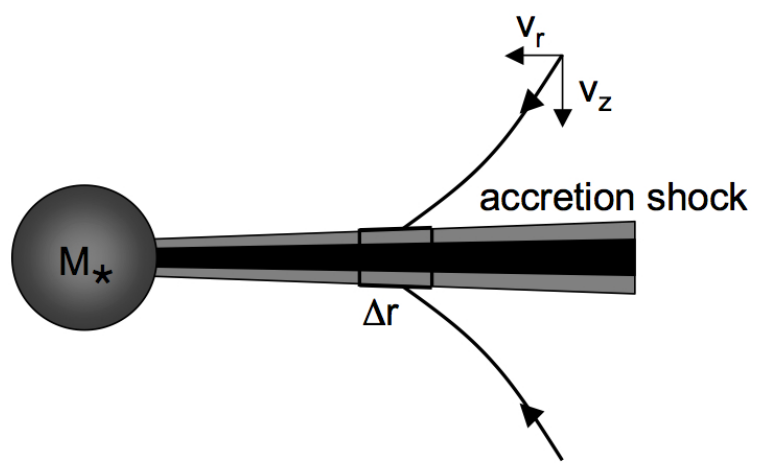

Figure 4. Schematic view of disk formation during the collapse of a rotating spherical cloud. Matter is falling toward the disk on mirrored path from locations above and below the midplane. The matter is stopped suddenly above the disk with an accretion shock that dissipated the free-fall energy.

Sect. 2 describes how a flat disk forms during the collapse of a rotating cloud. Since the simple collapse problem outlined there is spherically symmetric and the rotation itself axisymmetric, the trajectories of infalling material from the top are mirrored at the equatorial plane. The infalling fluid elements, which follow parabolic trajectories, thus collide in the equatorial plane with matter coming from the other side of the cloud. An accretion shock forms where the material collides and this accretion shock extends over the entire centrifugal radius $r_{\mathrm{c}}$. If the excess heat energy from the shock can be efficiently radiated away, a thin disk structure forms in the equatorial plane (Fig. 4).

The disk is supported against gravity by its rotation, but also to a lesser extent by the gas pressure gradient. To first order we can thus approximate the orbital velocity of the gas by the Keplerian orbital speed

$$
v_{\mathrm{K}}=\Omega_{\mathrm{K}} r=\sqrt{\frac{G M_{*}(t)}{r}} .
$$

We call $\Omega_{\mathrm{K}}$ the Kepler frequency

$$
\Omega_{\mathrm{K}}=\sqrt{\frac{G M_{*}(t)}{r^{3}}}
$$

At different stages of the evolution, $M_{*}(t)$ may not yet be the full mass of the star.

In the shock and subsequent cooling process, the vertical component of the infalling velocity is dissipated, so that the material within the disk is left with the parallel component of its infalling velocity $v_{r}$ (see Fig. 4). However, this remaining velocity is not necessarily equal to the Keplerian velocity at the point where the material falls onto the disk. This causes some additional mixing and angular momentum transport within the disk upon until its angular momentum and mass is redistributed in such a way that we end up with a disk in nearly Keplerian rotation. The point at which the infalling material hits the disk depends entirely on its initial angular momentum, i.e. its position within the cloud. 


\section{Disk structure}

For deriving the general equations of disk structure, we follow the motion of an annulus of the disk and use the vertically integrated form in all equations, i.e. the surface density $\Sigma$ instead of the physical density $\rho_{\text {gas }}$. Matter can flow into the annulus and out of it with a velocity $v_{r}$. This picture contains the implicit assumption that vertical motions in the disk can be neglected $\left(v_{z}=0\right)$ and that the radial motion is the same at all heights $\left(v_{r}(r, z)=v_{r}(r)\right)$.

We consider in the following an axisymmetric disk and assume that the surface density can be written in the general form of $\Sigma(r, t)$, which is an integral of the mass density $\rho_{\text {gas }}(r, z)$ over the disk height $z$

$$
\Sigma(r, t)=\int_{-\infty}^{\infty} \rho_{\text {gas }}(r, z) \mathrm{d} z .
$$

Then the vertically integrated continuity equation reads as

$$
r \frac{\partial \Sigma}{\partial t}+\frac{\partial}{\partial r}\left(r \Sigma v_{r}\right)=0
$$

and the vertically integrated conservation of orbital angular momentum becomes

$$
r \frac{\partial}{\partial t}\left(r^{2} \Omega \Sigma\right)+\frac{\partial}{\partial r}\left(r^{2} \Omega \cdot r \Sigma v_{r}\right)=\frac{1}{2 \pi} \frac{\partial \tau}{\partial r} .
$$

The two velocity components are $v_{r}$ and $v_{\Phi}=r \Omega$. The angular momentum of a thin annulus of the disk is $2 \pi r \Delta r \sum r^{2} \Omega$, where $\Delta r$ is the width of the annulus. The term on the right hand side of Eq. (13) arises from the viscous torques in the disk.

We briefly revisit here the concept of torque. A force that acts on the mass center of a body will cause a straight motion. If the same force acts on a point off the mass center, the body will start to rotate as well. The torque $\tau$ is then the product of the force $F$ and the lever, i.e. the distance from the mass center $r$

$$
\tau=r F \sin \theta
$$

where $\theta$ is the angle between the force vector and the lever vector. The accretion disk is not rotating as a solid body and so we can apply the concept of torque on each annulus in the disk. The neighbouring annulus will exert a force on it proportional to the difference in orbital velocities, the orbital velocity gradient $\mathrm{d} \Omega / \mathrm{d} r$. The force is called the shear force or viscous force.

The rate of shearing due to the orbital velocity gradient is $r \mathrm{~d} \Omega / \mathrm{d} r$. which has the units of $\mathrm{s}^{-1}$. Without describing yet the details of the viscosity or angular momentum transport, we note that

$$
\tau=2 \pi r \cdot v \Sigma r \frac{\mathrm{d} \Omega}{\mathrm{d} r} \cdot r=2 \pi r^{3} v \Sigma \frac{\mathrm{d} \Omega}{\mathrm{d} r} .
$$

Here, $v$ is the kinematic viscosity and $\tau$ is the torque exerted on an annulus in the disk. It is the product of the circumference, the viscous force per unit length $(v \Sigma A=v \Sigma r \mathrm{~d} \Omega / \mathrm{d} r)$ and the lever $r$.

Using the expression for the torque $\tau$, we can rewrite Eq. (13) as

$$
\frac{\partial}{\partial t}\left(r^{2} \Omega \Sigma\right)+\frac{1}{r} \frac{\partial}{\partial r}\left(r^{2} \Omega \cdot r \Sigma v_{r}\right)=\frac{1}{r} \frac{\partial}{\partial r}\left(v \Sigma r^{3} \frac{\mathrm{d} \Omega}{\mathrm{d} r}\right) .
$$

We can now use the continuity equation (Eq. 12) to eliminate the time dependence and simplify this to

$$
\Sigma v_{r} \frac{\partial\left(r^{2} \Omega\right)}{\partial r}=\frac{1}{r} \frac{\partial}{\partial r}\left(v \Sigma r^{3} \frac{\mathrm{d} \Omega}{\mathrm{d} r}\right) .
$$


The derivatives of $\Omega$ can be worked out, if we assume that the orbital frequency equals the Kepler frequency $\Omega_{\mathrm{K}}$. Then, this equation yields an expression for the radial velocity in the disk

$$
v_{r}=-\frac{3}{\Sigma \sqrt{r}} \frac{\partial}{\partial r}(v \Sigma \sqrt{r}) .
$$

Alternatively, we can insert Eq. (17) into Eq. (12) to eliminate $v_{r}$ and obtain

$$
\frac{\partial \Sigma}{\partial t}=\frac{1}{r} \frac{\partial}{\partial r}\left[\left(\frac{\mathrm{d}}{\mathrm{d} r}\left(r^{2} \Omega\right)\right)^{-1} \frac{\partial}{\partial r}\left(v \Sigma r^{3}\left(-\frac{\mathrm{d} \Omega}{\mathrm{d} r}\right)\right)\right] .
$$

If we assume again that $\Omega=\Omega_{\mathrm{K}}$, we can simplify this to

$$
\frac{\partial \Sigma}{\partial t}=\frac{3}{r} \frac{\partial}{\partial r}\left[\sqrt{r} \frac{\partial}{\partial r}(v \Sigma \sqrt{r})\right] .
$$

\subsection{Steady state disk structure}

In steady state, the disk structure follows from radial and angular momentum conservation and the assumption that the vertical component of gravity from the star is balanced by the vertical gas pressure gradient (vertical hydrostatic equilibrium). We study for this a disk annulus at a distance $r$ from the star. Material can flow into and out of the annulus with the velocity $v_{r}$ in radial direction. The radial momentum conservation equation for a steady-state flow is

$$
v_{r} \frac{\partial v_{r}}{\partial r}-\frac{v_{\phi}^{2}}{r}+\frac{1}{\rho_{\text {gas }}} \frac{\partial P}{\partial r}+\frac{G M_{*}}{r^{2}}=0,
$$

where $v_{r}$ is the radial velocity of the gas, $v_{\phi}$ is the circular velocity, and $M_{*}$ is the mass of the central star. The gas sound speed is defined as $c_{s}^{2}=\partial P / \partial \rho_{\text {gas }}$, where $P$ is the gas pressure and $\rho_{\text {gas }}$ is the gas density. For an ideal gas, $c_{s}=\sqrt{k T_{g} /\left(\mu m_{\mathrm{p}}\right)}$. Here, $k$ is the Boltzmann constant, $T_{g}$ is the gas temperature, $\mu$ is the mean molecular weight of the gas, and $m_{\mathrm{p}}$ is the proton mass. The four terms in the above equation arise from radial mass flow, centrifugal force, gas pressure, and gravity. Since pressure typically decreases with increasing radius, the third term is nearly always negative; effectively, gas pressure resists the gravitational force, resulting in gas rotating at sub-Keplerian orbital velocities.

Following (Eq. 13), assuming steady-state (e.g. $\partial / \partial t=0)$ and using the expression for the torque $\tau$, the angular momentum conservation equation becomes

$$
\frac{\partial}{\partial r}\left(r v_{r} \Sigma r^{2} \Omega\right)=\frac{\partial}{\partial r}\left(v \Sigma r^{3} \frac{\mathrm{d} \Omega}{\mathrm{d} r}\right)
$$

where the left-hand side is the radial change in angular momentum and the right-hand side arises from the viscous torques. Integrating this equation yields the following expression

$$
v \Sigma \frac{\mathrm{d} \Omega}{\mathrm{d} r}=\Sigma v_{r} \Omega+\frac{C}{2 \pi r^{3}} .
$$

At the inner edge of the disk, the shear vanishes because the disk is truncated there. So at that point $\mathrm{d} \Omega / \mathrm{d} r=0$. We can use this to get an expression for the integration constant

$$
C=-2 \pi \Sigma v_{r} \Omega r^{3}=-2 \pi r \Sigma v_{r} r^{2} \Omega=\dot{M} r^{2} \Omega .
$$


Here, $\dot{M}=2 \pi r \Sigma\left(-v_{r}\right)$, because in an accretion flow, the radial velocity is inward and thus negative. For simplicity, we assume that the disk extends down to the star. Then, the shear vanishes close to the star for

$$
C=\dot{M} \sqrt{G M_{*} R_{*}} .
$$

The integration constant can thus be seen as the influx of angular momentum through the disk. With this and $\Omega=\Omega_{\mathrm{K}}=\sqrt{G M_{*} / r^{3}}$, the disk surface density becomes

$$
\Sigma(r)=\frac{\dot{M}}{3 \pi \nu}\left(1-\sqrt{\frac{R_{*}}{r}}\right),
$$

for radii much larger than the stellar radius. Note that the viscosity $v$ is not a constant in the equation, but $\dot{M}$ obviously is.

\subsection{Vertical disk structure}

The vertical (z-direction) disk structure is found by solving the equation of hydrostatic equilibrium,

$$
\frac{1}{\rho_{\mathrm{gas}}} \frac{\partial P}{\partial z}=\frac{\partial}{\partial z}\left(\frac{G M_{*}}{\sqrt{r^{2}+z^{2}}}\right) .
$$

For an ideal gas, pressure and density are connected by $P / \rho_{\text {gas }}=c_{s}^{2}=k T /\left(\mu m_{p}\right)$, which can be used to eliminate $P$. If the disk is vertically thin $(z \ll r)$ and the gas temperature does not depend on $z$ (i.e. a vertically isothermal disk), Eq. (27) can be directly integrated to give the vertical density structure,

$$
\rho_{\text {gas }}(r, z)=\rho_{c}(r) \mathrm{e}^{-z^{2} /\left(2 H_{\text {gas }}^{2}\right)},
$$

where $\rho_{c}(r)$ is the density at the disk midplane and the gas scale height is given by

$$
H_{\mathrm{gas}}=\sqrt{k T_{c} r^{3} /\left(\mu m_{p} G M_{*}\right)},
$$

where $T_{c}$ is the midplane gas temperature. This equation shows that the gas scale height is the ratio of the gas sound speed to the angular velocity $\left(H_{\mathrm{gas}}=c_{s} / \Omega\right)$. In reality, neither $T_{g}$ nor $\mu$ are constant with $z$, and this can lead to large changes in the density structure high up in the disk. However, since we are here concerned with the mass dominating part of the disk close to the midplane, we work with these simplifying assumptions.

\section{Radial disk structure}

Having found an expression for the scale height, we return to the radial disk structure. We parametrise the viscosity as $v=\alpha c_{s} H_{\text {gas }}$ (which will be introduced later in Sect. 5.2 after a detailed discussion of the viscosity in disks). Inserting this relation along with Eq. (29) into Eq. (26), and assuming $r \gg R_{*}$ we find

$$
\Sigma=\frac{\mu m_{p} \sqrt{G M_{\star}}}{3 \pi k} \frac{\dot{M}}{\alpha T_{c} r^{3 / 2}} .
$$

For a disk with a simple powerlaw midplane temperature profile, $T_{c} \propto r^{-q}$, the surface density is proportional to $r^{q-3 / 2}$, thus also a simple powerlaw of radius. This is a rather interesting results that results directly from accretion disk theory, under the assumption that $\alpha$ is a constant throughout the disk. 
Further, the midplane density may be written as

$$
\rho_{c} \simeq \Sigma / H_{\text {gas }}=\rho_{\text {in }}\left(\frac{r}{r_{\text {in }}}\right)^{\frac{3}{2} q-3},
$$

where $\rho_{\text {in }}$ is the density at the inner disk radius $r_{\text {in }}$. Assuming a simple powerlaw for the temperature profile is a first guess, but is there actually a way to determine the temperature profile within an accretion disk?

In an accretion disk, the energy generation is predominantly through viscous torques, $\tau$ (see also the discussion in the chapter on heating and cooling processes in disks, Woitke 2015). The net torque on a disk annulus with width $\Delta r$ is equal to the difference between the torque on the outer and inner surface

$$
\tau(r+\Delta r)-\tau(r)=\frac{\partial \tau}{\partial r} \Delta r
$$

The energy generated by this torque is then

$$
\Omega \frac{\partial \tau}{\partial r} \Delta r=\left[\frac{\partial}{\partial r}(\tau \Omega)-\tau \frac{\mathrm{d} \Omega}{\mathrm{d} r}\right] \Delta r
$$

The first term, when added up over the entire disk, is simply $\left.\tau \Omega\right|_{r_{\text {out }}}-\left.\tau \Omega\right|_{r_{\text {in }}}$ and thus given by the disk boundary conditions. The second term describes the local energy dissipation and thus the heat generation in an annulus in the disk. The energy generation per unit surface area is given by $\tau(\mathrm{d} \Omega / \mathrm{d} r) \Delta r /(2 \pi r \Delta r)$. We assume that this energy is not transported in the disk, but is radiated away locally through the two surfaces of the disk, with $\sigma T_{\text {disk }}^{4}$, where $T_{\text {disk }}$ is the effective temperature of the disk. Therefore we have (note the factor 2 due to the two sides of the disk)

$$
\sigma T_{\mathrm{disk}}^{4}=-\frac{\tau \mathrm{d} \Omega / \mathrm{d} r}{4 \pi r}=-\frac{1}{2} \nu \Sigma\left(r \frac{\mathrm{d} \Omega}{\mathrm{d} r}\right)^{2}=\frac{9}{8} \nu \Sigma \Omega^{2} .
$$

Here we have filled in the torque $\tau$ using Eq. (15). Also, we have assumed that the disk has a Keplerian rotation profile $\Omega=\sqrt{G M_{*} / r^{3}}$. Using the relation found between the mass accretion rate and the viscosity, $\dot{M}=3 \pi v \Sigma$ for $r \gg R_{*}$, we can eliminate the viscosity from this equation

$$
\sigma T_{\text {disk }}^{4}=\frac{3}{8 \pi} \dot{M} \Omega^{2}
$$

This yields a temperature profile of

$$
T_{\text {disk }}=\left(\frac{3}{8 \pi \sigma} \dot{M} \Omega^{2}\right)^{1 / 4},
$$

thus a $r^{-3 / 4}$ profile. We see that the disk temperature does not depend on the viscosity. The implicit assumption here is that the viscosity of the disk corresponds to its accretion rate, an observable quantity. Hence we do not need to know the details of the angular momentum transport to describe the disk structure. Using a relatively high observed accretion rate for a very young T Tauri $\operatorname{star}\left(M_{*}=1 \mathrm{M}_{\odot}\right)$ of $10^{-7} \mathrm{M}_{\odot} / \mathrm{yr}$, we obtain a disk temperature of $150 \mathrm{~K}$ at a distance of $1 \mathrm{AU}$, if the temperature is dominated by viscous heating. 


\section{Angular momentum transport}

The specific angular momentum, which is the angular momentum per unit mass, stored in a $1 \mathrm{M}_{\odot}$ disk with a size of $10 \mathrm{AU}$ is $3 \times 10^{53} \mathrm{~cm}^{2} / \mathrm{s}$. On the other hand, a $1 \mathrm{M}_{\odot}$ star rotating at break-up velocity has a specific angular momentum of $6 \times 10^{51} \mathrm{~cm}^{2} / \mathrm{s}$. Hence, the original angular momentum of the disk is 50 times higher than the maximum allowed for a star. Since angular momentum is strictly conserved, there needs to be a process that actually transports angular momentum away to prevent it from accumulating on the star. The main possibilities are a torque from the external medium (e.g. magnetic fields), viscosity inside the disk transporting angular momentum to the outer disk, and disk winds taking angular momentum away. In the following, we discuss the basic principle of angular momentum transport within the disk. Disks winds are certainly important as well, but their theory is complex and we ignore them in this course.

If we assume for a moment that the disk rotates with Keplerian speed and consider only two particles with masses $m_{1}$ and $m_{2}$, we can write their total (kinetic plus potential) energy $E$ and angular momentum $J$ as

$$
\begin{aligned}
E & =-\frac{G M_{*}}{2}\left(\frac{m_{1}}{r_{1}}+\frac{m_{2}}{r_{2}}\right) \\
J & =m_{1} v_{\Phi, 1} r_{1}+m_{2} v_{\Phi, 2} r_{2} \\
& =\sqrt{G M_{*}}\left(m_{1} \sqrt{r_{1}}+m_{2} \sqrt{r_{2}}\right),
\end{aligned}
$$

where $r_{1}$ and $r_{2}$ are the corresponding distances of the two masses from the central star with mass $M_{*}$. From the conservation of angular momentum of the total system, it follows that a small change in orbit for one of the masses requires a corresponding change for the other one

$$
\begin{aligned}
\frac{\partial J}{\partial r} & =-m_{1} \frac{1}{2 \sqrt{r_{1}}} \\
\Delta J & =\frac{\mathrm{d} J}{\mathrm{~d} r_{1}} \Delta r_{1}+\frac{\mathrm{d} J}{\mathrm{~d} r_{2}} \Delta r_{2}=0 \\
m_{1} \frac{\Delta r_{1}}{\sqrt{r_{1}}} & =-m_{2} \frac{\Delta r_{2}}{\sqrt{r_{2}}}
\end{aligned}
$$

The same of course holds true if we consider two neighboring annuli in the disk.

This process requires a way of connecting the two particles (or rings of material) with each other. So, what remains to be identified is the source of this coupling. We can consider for example the friction between the two annuli to be caused by their difference in rotation speed (shear motion). However, since we deal here with a gas, diffusion plays a role in transporting gas in both directions, inward and outward. We can hence consider small turbulent random motions as a cause of radial mixing of material and thus as a cause of coupling the various annuli with the disk.

\subsection{Turbulent viscosity}

We can derive a simple estimate of the molecular viscosity $v_{m}$ through

$$
v_{m} \approx \lambda c_{s}
$$

where $\lambda$ is the mean free path of the molecules given by $1 /\left(n \sigma_{m}\right)$, the product of gas particle density $n$ and collisional cross section $\sigma_{m}$ between the molecules. If we approximate the latter by the typical 
physical size of molecules $\left(2 \times 10^{-15} \mathrm{~cm}^{2}\right)$, we obtain for a typical distance of $10 \mathrm{AU}$ in the disk a molecular viscosity of

$$
v_{m}=\frac{1}{n \sigma_{m}} c_{s}=\frac{5 \times 10^{4}}{10^{12} \times 2 \times 10^{-15}} \mathrm{~cm}^{2} / \mathrm{s}=2.5 \times 10^{7} \mathrm{~cm}^{2} / \mathrm{s} .
$$

If we estimate the corresponding viscous timescale $t_{v}$ for radial transport at $r=10 \mathrm{AU}$

$$
t_{v}=\frac{r^{2}}{v_{m}} \approx 3 \times 10^{13} \mathrm{yr}
$$

we immediately see, that we can rule out molecular viscosity as the main source of friction in these accretion disks. The typical disk evolutionary timescale is of the order of a few million years, so more than ten million times shorter than this molecular viscosity timescale.

The Reynolds number is used in gas dynamics to describe the ratio of inertial forces (resistance to change or motion) to viscous forces (glue) and thus also to define whether a fluid is laminar or turbulent. The Reynolds number Re of a typical accretion disk can be estimated as

$$
\operatorname{Re}=\frac{V L}{v_{m}},
$$

where $V$ and $L$ are characteristic velocity and length scales in the disk, i.e. $V=c_{s}=0.5 \mathrm{~km} / \mathrm{s}$ at $10 \mathrm{AU}$ and $L=H_{\text {gas }}=0.05 r=0.5$ AU. Filling in these numbers, we obtain a Reynolds number of $\operatorname{Re} \approx 10^{10}$. Hence, in the presence of some instability, the disk is highly turbulent. The Reynolds number also indicates the ratio between the largest and smallest scales of fluid motion (sometimes referred to as eddies). The largest scale fluid motion is set by the geometry of the disk (e.g. its scale height), while the smallest scales are here a factor $\sim 10^{10}$ smaller.

\subsection{Shakura-Sunyaev viscosity}

If molecular viscosity is not large enough to provide a source of turbulence that drives the angular momentum transport in disks, what else can it be? We need to identify possible instabilities that can cause turbulent friction larger than that due to molecular viscosity. Before we go into that discussion, we present here a very successful idea of parametrising the viscosity without identifying its source. The idea goes back to Shakura \& Sunyaev (1973) who first proposed the so-called $\alpha$ parameter to describe the efficiency of angular momentum transport.

Since the largest turbulent scales will be set by the geometry of the flow, we can use the vertical scale height of the gas as a representative scale. Along the same dimensional analysis scheme, we can use the sound speed $c_{s}$ as the characteristic velocity of the turbulent motions. Hence, we can write the viscosity $v$ as

$$
v=\alpha c_{s} H_{\text {gas }} .
$$

We can now express the viscosity in terms of the disk parameters and hence estimate the efficiency of angular momentum transport and thus mass accretion onto the central star. It is also easy to estimate how large $\alpha$ should be to reproduce the observed timescale of disk evolution. The viscous timescale $\tau_{\text {vis }}$ can be expressed as

$$
\tau_{\mathrm{vis}}=\frac{r^{2}}{v}=\left(\frac{H_{\mathrm{gas}}}{r}\right)^{-2} \frac{1}{\alpha \Omega} .
$$

Here, we can fill in 1 Myr as the typical evolutionary timescale at 50 AU. In addition, we assume that the disks are indeed very thin and $H_{\text {gas }} / r \sim 0.05$. This yields an $\alpha$ of 0.02 . 

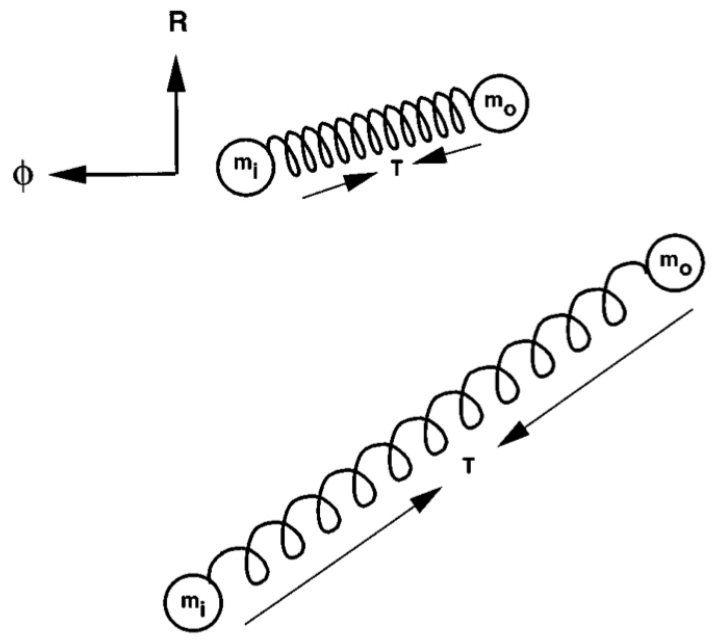

Figure 5. Two masses in orbit connected by a weak spring. The spring exerts tension force $T$ resulting in a transfer of angular momentum from the inner mass $m_{i}$ to the outer mass $m_{o}$. If the spring is weak, the transfer results in an instability as $m_{i}$ loses angular momentum, drops through more rapidly rotating inner orbits, and moves further ahead. The outer mass $m_{o}$ gains angular momentum, moves through slower outer orbits, and drops further behind. The spring tension increases and the process runs away (figure and caption from Balbus \& Hawley 1998).

\subsection{MRI}

In the presence of magnetic fields, the field lines act like springs connecting different annuli within the disk. Fig. 5 illustrates the basic principle. If a weak pull exists between two masses in adjacent annuli of the disk, $m_{i}$ and $m_{o}$, the inner mass element will loose angular momentum and hence drift even further inward, while the outer mass element gains angular momentum and drifts further outward. The new orbital velocity of the inner mass element is even higher than before (presuming that $\Omega$ decreases with $r$ ), while the new orbital velocity of the outer element is even smaller. This effect thus enhances the velocity difference between the two masses in adjacent annuli, giving rise to an instability. The instability is generally referred to as magneto-rotational instability and has been described first by Balbus \& Hawley (Balbus \& Hawley (1998) - hence we often refer to it as Balbus-Hawley instability). A prerequisite for a magnetised disk to be linearly unstable is that the orbital velocity decreases with radius

$$
\frac{\mathrm{d}}{\mathrm{d} r}(\Omega)<0
$$

a condition clearly satisfied in Keplerian disks. In addition, the disk needs to be ionised to a certain degree since neutral gas does not couple efficiently to the magnetic field lines. The critical ionisation degree is $n_{e} / n_{\text {tot }} \sim 10^{-12}$ to sustain turbulence generation by the magneto-rotational instability (Sano $\&$ Stone 2002).

It is very difficult to assess the amount of viscosity generated by MRI. The only way of measuring $\alpha$ would be through numerical magneto-hydrodynamical simulations. Disentangling physical and 
numerical effects becomes then a challenge. However, from simulations it seems that $\alpha$ could be consistent with a value of $\sim 10^{-2}$ as inferred from observations.

Acknowledgements The author is indepted to Kees Dullemond who once explained the basic physics of viscous accretion disks to him in just half an hour. The research leading to these results has received funding from the European Union Seventh Framework Programme FP7-2011 under grant agreement no 284405 .

\section{References}

Balbus, S. A. \& Hawley, J. F. 1998, Reviews of Modern Physics, 70, 1

Beckwith, S., Sargent, A. I., Scoville, N. Z., et al. 1986, ApJ, 309, 755

Koerner, D. W., Sargent, A. I., \& Beckwith, S. V. W. 1993, Icarus, 106, 2

Mannings, V., Koerner, D. W., \& Sargent, A. I. 1997, Nature, 388, 555

O’Dell, C. R., Wen, Z., Hu, X., \& Hester, J. J. 1992, in Bulletin of the American Astronomical Society, Vol. 24, American Astronomical Society Meeting Abstracts, 1147

Rodriguez, L. F., Canto, J., Torrelles, J. M., Gomez, J. F., \& Ho, P. T. P. 1992, ApJL, 393, L29

Sano, T. \& Stone, J. M. 2002, ApJ, 570, 314

Sargent, A. I. \& Beckwith, S. 1987, ApJ, 323, 294

Shakura, N. I. \& Sunyaev, R. A. 1973, A\&A, 24, 337

Shu, F. H. 1977, ApJ, 214, 488

Woitke, P. 2015, in EPJ Web of Conferences, Vol. 102, Summer School on Protoplanetary Disks: Theory and Modeling Meet Observations, ed. I. Kamp, P. Woitke, \& J. D. Ilee 